\title{
Die Rationalisierung der Bewegung bei Gottfried Wilhelm Leibniz
}

\author{
Raphael Borchers \\ Universität Leipzig \\ Institut für Philosophie
}

\begin{abstract}
Zusammenfassung
Der Begriff der Bewegung ist unabdingbar in jeder Analyse von Weltereignissen. Aber Überlegungen über die Bewegung selbst kamen zwei Jahrtausende nicht ohne den Rückgriff auf okkulte Kräfte in der Natur aus. Erst Galileo Galilei gelingt qua more geometrico ein Verständnis der Nomologizität von Bewegungsprozessen mit bis dato unerreichten mechanizistischen Anwendungserfolgen. Gottfried Wilhelm Leibniz hingegen insistiert vor diesem Hintergrund auf traditionelle Einsichten, um einem rationalen Verständnis der Bewegung Genüge $\mathrm{zu}$ tun, ohne freilich Galileis geometrischen Standpunkt zurück zu weisen. Er postuliert eine Rationalisierung der Bewegung, die über die bloß geometrische Perspektive hinaus gehen müsse, um den ultimativen Grund der Bewegung einsichtig $\mathrm{zu}$ machen. Auf den galileischen Prinzipien aufbauend und in intensiver Auseinandersetzung mit der cartesianischen Phoronomie führt ihn dieser Anspruch letztlich zur Wissenschaft der Dynamik als einer ,Logik des Lebens'.
\end{abstract}

\section{Was ist Bewegung?}

Der Begriff der Bewegung ist fundamental für die Wissenschaft, zumal für die Naturwissenschaft. Wer in Un-

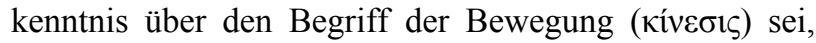
konstatiert Aristoteles in seine Abhandlung über die Bewegung im dritten Buch der Physik (200b12-15), der sei es notwendig auch im Hinblick auf die ganze Natur. Hierin pflichtet ihm der vermeintliche Anti-Aristoteliker und Begründer der modernen Mechanik, Galileo Galilei, bei, wenn er den dritten Tag seines berühmten Discorsi mit einer ganz ähnlichen Bemerkung beginnen lässt: Nichts in der Natur, konstatiert er dort, sei älter als die Bewegung (Ed. Naz. VIII: 190). Wenngleich Galilei hier darauf abhebt, dass es sich beim Begriff der Bewegung um einen der am meist bedachtesten Gegenstände der Philosophie handelt, zu dem er dennoch neue und überaus wichtige Einsichten hinzufügen könne, ist hiermit außerdem zum Ausdruck gebracht, dass alle Natur nichts wäre, wäre sie nicht bewegt und dass wir nichts von ihr

Erschienen in: arbeitstitel - Forum für Leipziger Promovierende, Band 8, Heft 1 (2020), S. 2- 9. Lizenzierung CC-BY-SA 4.0. wüssten, würden wir die Bewegung nicht zur Grundlage unserer Analyse machen.

So leicht diese Feststellung aber auch fällt und so selbstverständlich uns Begriffe wie der der Bewegung erscheinen, so schwer ist es für gewöhnlich bei dieser Art von Fundamentalbegriffen sie zufriedenstellend $\mathrm{zu}$ bestimmen. Sie sind uns allzu usitatus et abditus - alltäglich und doch so verborgen, wie es Augustinus in jener bekannten Meditation über die Zeit im elften Buch seiner Confessiones (XI.14/17, 22/28) beklagt hat, die mit der hier vorgelegten Frage nach der Bewegung eine natürliche Verwandtschaft aufweist. Bewegung ist uns gleichfalls eine Selbstverständlichkeit solange wir uns nur nicht fragen, was sie eigentlich ist. Die Schwierigkeit sowohl mit Blick auf den Begriff der Zeit wie den der Bewegung liegt darin, „dass es sich um keinen Gegenstand der Vorstellung handelt" $[=$,,motus non esse rem subjectam imaginationi“"] (AA VI.3: 531) auf den er sich bezieht. $\mathrm{Zu}$ dieser Feststellung kommt der junge und wissbegierige Soldat Charinus in Leibniz' frühen Dialog über die Bewegung (Pacidius Philalethi, AA VI.3: 528-571). Was wir uns vor-stellen, bestätigt ihm auch sein Dialogpartner Guilielmi Pacidius, ist uns vor unsere äußeren Sinne gestellt. In diesem Sinne sei die Bewegung niemals in unserer empirischen Anschauung. Was wir mit unseren äußeren Sinnen anschauen, wenn wir der Bewegung gewahr werden, ist stets das Bewegte, nicht die Bewegung.

Aus der bloßen Vorstellung des Bewegten stammt daher auch die naive Bestimmung des Charinus', dass Bewegung doch nichts anderes sei als die Ortsveränderung eines Körpers in der Zeit. Bitter beschwert er sich dann allerdings darüber, dass ihn Pacidius - hinter dem sich offenkundig der Autor selbst verbirgt - verspotten wolle, wenn dieser bekennt, dass ihm zum Verständnis dieser Bestimmung bedauerlicherweise die geistigen Kapazitäten abgingen (AA VI.3: 534 f.; GM VI: 237). Gewiss ist die sokratische Ironie in dieser Erwiderung nicht $\mathrm{zu}$ übersehen, die Charinus sehr in Verlegenheit bringt. Doch Pacidius insistiert ganz zu Recht darauf, dass seine Ironie nicht der scholastischen Überheblichkeit eines Philosophen über einen physikalischen Geometer geschuldet sei, sondern dass mit der Antwort des Charinus die tatsächlich bestehenden Schwierigkeiten der Frage nach der Bewegung noch gar nicht berührt seien. Diese gelte es in das Blickfeld zu rücken, wenn man denn gewillt sei, sich dieser Frage anzunehmen. Das hieße zwar keineswegs, dass Charinus' Antwort eine schlechte 
oder gar falsche Antwort ist, aber sie sei eben doch bloß eine symbolische, wie sich Leibniz in seiner späteren Terminologie der Meditationes de Cognitione, Veritatae et Ideis (AA VI.4: 585-592) ausdrücken wird. Eine symbolische bzw. suppositiven Erkenntnis unterscheide sich von einer intuitiven bzw. kontemplativen dadurch, dass sie bei der Analyse der Identität eines Begriffs gewisse Annahmen trifft, die sie explizit oder implizit von der Analyse ausnimmt, während letztere die Analyse vollständig durchzuführen in der Lage sei.

\section{Kinesis und Phora}

$\mathrm{Zu}$ den impliziten Voraussetzungen unserer alltäglichen Redeweise von der Bewegung ist jene bereits erwähnte begriffliche Trennung des Bewegten von dem Bewegenden zu zählen. In guter Tradition wird trivialerweise unterschieden zwischen dem, das bewegt wird, und dem, das das Bewegte bewegt. Von diskutablen philosophischen Interesse ist dabei jener Fall, bei dem beides in Eins zusammenfällt. Dieser Fall ist relativ selten, manche meinen ein Hirngespinst (Hobbes), andere meinen, er sei absolut singulär, aber gleichwohl notwendig (Descartes). Man nennt diesen Fall des Bewegtseins durchaus treffend eine Selbst-Bewegung. Üblicher hingegen ist uns die Redeweise, dass etwas ,animiert' oder ,lebendig' ist. Ein ,Animal' ist nach dem traditionellen Verständnis etwas, das eine Seele [= anima] als Grund ihrer Selbstbewegung besitzt und wird im Deutschen mit ,Tier', das lateinisch die bestia ist, eher schlecht übersetzt.

Traditionelle Erklärungen, die auf das Konzept einer solchen intrinsischen Disposition zur Selbstbewegung, einem Bewegungsgrund im Wesen des Bewegten selbst zurückgriffen, wurden im Zuge der Entwicklung moderner Wissenschaft im 16. und 17. Jahrhundert fraglich und mit Rekurs auf eine damals sehr geläufige Ausdrucksweise als „,qualitates occultae “1 verspottet. Die Annahme geheimer Qualitäten, die bewirken, dass etwa ein Stein nach unten und nicht nach oben fällt, wie es in einem ironischen Witz heißt, wurde zum unwissenschaftlichen Aberglauben, den es zu überwinden galt. Dem prosaischen Postulat Galileis im Il Saggiatore, dass die Natur in der Sprache der Mathematik geschrieben und ihre Zeichen Dreiecke, Kreise und andere geometrischen Figuren seien (Ed. Naz. VI: 232), entsprach der weitaus üblichere Fall des Bewegtseins sehr viel besser. Er unterscheidet sich von der Selbstbewegung dadurch, dass das Bewegte und Bewegende grundsätzlich auseinanderfällt und sich die Analyse der Bewegung auf die geometrische

${ }^{1}$ Vgl. zum Begriff der okkulten Formen die einschlägigen Stellen bei Thomas v. A. in De operationibus occultis naturae und Summa contra Gentiles II.68, III.92. Der Gebrauch von ,forma occulta', ,qualitas occulta', ,proprietas occulta ${ }^{6}$ oder ,vir occultus' ist im 17. Jahrhundert bei sämtlichen Autoren, so auch bei Leibniz, pejorativ besetzt; vgl. hierzu etwa im Discours de Métaphysique $§ 10$ (AA VI.4: 1543) und die Rede von „qualitates óp’óñ 1706 (GP II: 294); bei Descartes vgl. Principia Philosophia IV, $\S 187$ (AT VIII.1: 314) und Meditationes de Prima Philosophia, Responsio ad sextas objectiones (AT VII: $442 \mathrm{f}$.); bei Bacon Novum Organum I, § 66 (1999: 137-141) und bei Hobbes Leviathan II.46 (1651: 375).
Analyse des Bewegten beschränkt. Ihn könnte man das Bewegt-Werden nennen, unglücklicher ist im Deutschen manchmal auch von einer Fremd-Bewegung die Rede. Damit wäre zum Ausdruck gebracht, dass die übliche Art des Bewegtseins in gewisser Weise passiv ist und erlitten oder ertragen wird, während die Selbstbewegung ganz allgemein ausgedrückt eine aktive Vollzugsform darstellt, die sich an sich oder an anderem vollzieht. Diese Unterscheidung - so trivial sie auch scheinen mag - ist nach Leibniz einer der fundamentalsten Grundsätze unseres Wissens und wissenschaftlichen Selbstverständnisses; womit sie nicht mehr bloß für die Naturwissenschaften, sondern ebenso für die Geisteswissenschaften - wenn man dieser Unterscheidung (noch) etwas abgewinnen kann oder will - von größter Relevanz wäre.

Auf diese grundlegende Bedeutung hingewiesen hat schon Platon in zwei prominenten Passagen über die Unsterblichkeit der Seele in den Dialogen Phaidros und Nomoi. Dort will zum einen Sokrates und zum anderen ein unbekannter Athener die bloße Kinetik von einer $A u$ tokinetik unterschieden wissen (Phdr. 245e, Nom. 894d896d; vgl. außerdem Charm. 168e, Polit. 269e sowie Aristoteles, Phys. 254b10-12, $261 \mathrm{a} 25$ f.). Jener Unbekannte in den Nomoi - hinter dem sich wohl Platon selbst verbirgt - weißt nach einem Abriss von acht verschiedenen Bewegungsarten darauf hin, dass es sich bei der Unterscheidung von Selbstbewegung und BewegtWerden um die grundlegendste und wichtigste handle, weil mit ihr die Frage nach dem Ursprung der Bewegung und damit die Frage nach ihrem Anfang aufgeworfen sei.

Worauf Platon mit der besonderen Betonung dieser Unterscheidung abhebt, das ist, dass eine Bewegung ganz generell unter zwei verschiedenen Gesichtspunkten betrachtet werden kann. Zum einen unter dem Aspekt, wie sich etwas bewegt, und zum anderen warum es sich bewegt. Bei der bloßen Betrachtung des Wie einer Bewegung bleibt die Frage nach dem Warum einer Bewegung notwendig außen vor. Es wird immer schon vorausgesetzt, dass es sich bewegt. Auf die Frage, warum sich überhaupt etwas bewegt, sind nach Platon zwei Antworten möglich: Erstens, weil das Bewegte durch etwas anderes bewegt wird oder wurde und damit blo $\beta$ kinetisch ist, und zweitens, weil es sich selbst bewegt oder bewegt hat und damit autokinetisch ist.

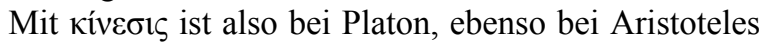
und der gesamten peripatetischen Tradition, über die Frage nach dem bloßen Wie einer Bewegung hinaus immer auch schon eine Perspektive eingenommen, die die Frage nach dem Grund einer Bewegung mit in den Blick nimmt. Die bürgerliche Bezeichnung, Kinematik' für die Analyse des bloßen Bewegungsablaufs ist daher nicht ganz unproblematisch, da mit kíveors traditionell jeglicher „Vollzug der Verwirklichung eines Möglichen“ gemeint ist, wie es bei Aristoteles heißt (Phys. $201 \mathrm{~b} 10$ f.; Metaphy. 1065b15-17). Was wir heute Kinematik nennen, blendet aber gerade jenen Gesichtspunkt des Warum, die Frage nach dem Beweggrund aus. Für Leibniz, der die eingangs angeführte zentrale Bedeutung der Bewegung für das wissenschaftliche Verständnis von Weltereignissen selbstverständlich teilt (GM VI: 237), ist dies die für eine selbstbewusste Wissenschaft unabdingbare 
Perspektive auf die ratio der Bewegung.

,Ratio' gebrauchen wir heute völlig richtig im Sinne von Vernunft. In Gegenüberstellung zu ,Ursache` [= causa] gebraucht Leibniz diesen Term jedoch auch noch (bzw. wieder) im allgemeinen Sinne der Bedingung für die noetische Anerkennung einer propositionalen Geltung. So ist in den meisten Formulierungen seines berühmten principium rationis sufficientis [= Satz des zureichenden Grundes] nicht von einer ,suffizienten Ursache' die Rede, sondern von einem ,suffizienten bzw. determinierenden Grund'. In seinem Kern besagt dieser Satz daher nicht, dass alles bloß Effekt einer kausalen Verkettung sei, wie es immer wieder missverstanden wurde und wird, sondern dass alles, was gewusst werden kann, sich auch zureichend begründen lassen können muss; ${ }^{2}$ was einen ganz wesentlichen Unterschied zum fatalistischen Verständnis macht, das Leibniz bis heute in den Mund gelegt wird.

An diese leibnizsche Bestimmung angelehnt sei im Folgenden von einer Rationalisierung die Rede. Rationalisiert ist eine Proposition nach Leibniz dann, wenn ein Grund ,erfunden ' werden kann, der hinreicht, ihre Geltung (an)zu-erkennen. ${ }^{3}$ Dieser Grund könne aufgrund einer harmonischen Parallelität des Reichs der Natur und des Reichs der Moral zwar durchaus eine lokale Ursache sein, aber gerade die Lokalität dieser Ursache verhindert, dass sie je als Grund zureicht (GP IV: 391, VI: 113, 142; GM VI: $242 \mathrm{f}$.). Mit anderen Worten: Eine ,gleichgewichtige Indifferenz' [=,indifference d'équilibre" $]$ zwischen zwei Ereignismöglichkeiten, den reinen und tatsächlichen $\mathrm{Zu}-\mathrm{Fall}$ [= „causalité absolute et réelle"] zur einen oder anderen Seite hin gibt es nach Leibniz nicht (GP VI: 296 f.). Alles ließe sich mehr oder weniger rationalisieren, sofern wir den Anspruch erheben, überhaupt etwas wissen zu können. Die reine Kontingenz der Ereignisse widerspreche vielmehr dem wissenschaftlichen Selbstverständnis ebenso sehr wie die ,nackte Notwendigkeit' [= ,brutae necessitatis“ $]$ (GP IV: 391; AA VI.4: 1575-1578). Leibniz' Überlegungen zu einer Rationalisierung wissenschaftlicher Begriffe gleicht mithin der waghalsigen Schifffahrt zwischen Skylla und Charybdis als einem allzu voreiligen Skeptizismus

\footnotetext{
${ }^{2}$ Die von Leibniz ebenfalls gebrauchte Formulierung „Nihil est sine causa" $[=$ „Nichts ist ohne Ursache" $]$ in Catena Mirabilium Demonstrationum de Summa Rerum (AA VI.3: 584), die eindeutig an die frühe Formulierung des Satzes vom Grunde ,Nihil est sine ratione" [= ,Nichts ist ohne Grund" $]$ in z. B. Demonstratio Propositionum Primarum (AA VI.2: 479) angelehnt ist und sich auch in der Confessio Philosophi (AA VI.3: 126) wiederfindet, bedürfte hier einer eingehenden Erläuterung. Zusammengefasst gilt es hier jedoch zu beachten, dass in der zitierten Passage explizit auf die Kraft-Äquivalenz von Ursache und Wirkung abgehoben wird, die selbstredend nicht ohne die causa efficiens auskommt. Zu beachten ist außerdem die nach Leibniz mit causa und ratio einhergehende Perspektivendifferenz der mit- und ineinander verschränkten $\mathrm{Ob}$ jekt- und Metaebene wissenschaftlicher Untersuchungen.

${ }^{3}$ Leibniz spricht von einer ,Kunst des (Gründe-)Erfindens ${ }^{6}$ [=ars inveniendi $]$ als der geeigneten Rationalisierungsmethodik; vgl. hierzu etwa den Discours touchant la methode de la certitude etc. (AA VI.4: 951-204) sowie Projet et Essais pour avencer l'art d'inventer (AA VI.4: 963-970).
}

gegenüber allem Unbekannten und Unheimlichen, der sich deswegen des Blicks über den erhellten Fokus seiner Linse hinaus enthält, und einem bornierten Dogmatismus charakterisiert, der die Grenze des Selbstverständlichen unnötig sakralisiert und damit gleichfalls der wahrhaft wissenschaftlichen Betrachtung eine unnötige Grenzziehung auferlegt.

Eine im Hinblick auf diese rationale Perspektive ,nackte' Kinematik aber behandelt also die Bewegung sozusagen als irrationale Tatsache und setzt sie als eine (noch) nicht rationalisierte Weltbegebenheit voraus, da in ihr die Frage nach dem Grund dafür, dass sich Körper überhaupt bewegen, ausgeklammert wird; was freilich kein Problem ist, sofern man sich des symbolischen Charakters seiner darauf aufbauenden Analysen des Bewegten bewusst ist. Die bloß symbolische Behandlung der Bewegung, wie ich sie eingangs anhand von Charinus' vorschneller Antwort eingeführt hatte, betrachtet mithin ,die Bewegung als ein reines Quantum nach seiner Zusammensetzung ohne alle Qualität des Beweglichen“, wie es Immanuel Kant in der einschlägigen Passage seiner Metaphysischen Anfangsgründe der Naturwissenschaft formulierte (AA IV: 477). Allerdings spricht Kant dort noch nicht von Kinematik, sondern greift auf den heute gänzlich aus der Mode gekommenen Neologismus ,Phoronomie' zurück. ${ }^{4}$ Dieser war im 18. Jahrhundert vor allem durch das gleichnamige mechanische Hauptwerk des Schweizer Mathematikers Jakob Hermann (1716) bekannt, das von Leibniz gegen Ende seines Lebens noch aktiv gefördert und ausdrücklich gelobt wurde. Meiner Meinung nach ist diese Bezeichnung durchaus treffender für diejenige Disziplin, die das BewegtWerden, den bloßen Bewegungsprozess behandelt.

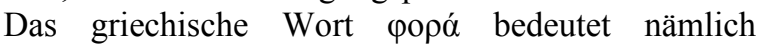
zunächst einmal so viel wie Bestattung, Abgabe, Steuer und Bezahlung, worin jedoch bereits zum Ausdruck kommt, dass etwas bezeichnet ist, das einigermaßen unfreiwillig mit einem geschieht, wozu man gewissermaßen von etwas Fremden außer sich genötigt wird. Abgeleitet

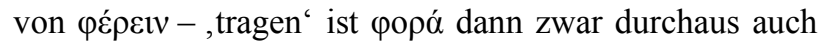
im Sinne von (Orts-)Bewegung zu verstehen - und bei Aristoteles etwa findet sich auch genau diese Verwen-

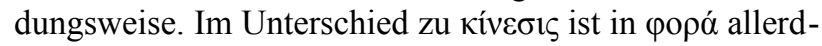
ings die Passivität des Bewegtseins betont, womit immer schon darauf hingewiesen wäre, dass es sich um eine ,ertragene' Bewegung handelt. Es wird hierbei in Kants Worten von der Qualität des Beweglichen abgesehen. Noch genauer könnte man vielleicht sagen, dass von den Eigenschaften des Trägers der Bewegung abgesehen und allein das betrachtet wird, was von ihm getragen wird; so wie der Inhalt einer Amphore unabhängig von dieser betrachtet werden kann, gleichwohl es ihn ohne seiner Trägerin als solchen gar nicht geben würde. Während also kíveбıs immer auch den Grund der Bewegung mit

${ }^{4}$ Über diesen Titel wurde bereits im Physikalischen Wörterbuch von Johann S. T. Gehlers (1833: 472 f.) gespottet, dass er zwar noch von manchen Philosophen gebraucht werde, sich aber ,in eigentlich physikalischen und mathematischen Werken selten oder nie" finden lasse. Im Wörterbuch der philosophischen Begriffe von Friedrich Kirchner et al. (1998) findet sich dann auch überhaupt kein Eintrag mehr. 
einbezieht, wird mit popá und nach Kant auch mit ,Phoronomie' auf die reine Nomologizität des Bewegungsablaufs in Raum und Zeit $[=$,motus vestigiis in spatio et tempore"] (Leibniz 1906: 115) abgehoben.

\section{Phoronomie oder Logik der Physik}

Entgegen der geläufigen Annahme (Mainzer 1984) geht die Bezeichnung ,Phoronomie' jedoch nicht auf Jakob Hermann und auch nicht auf Leibniz selbst zurück. Vor ihnen betitelte bereits der für seine terminologische Verspieltheit bekannte Lübecker Logikdidaktiker und Entomologe Joachim Jungius eine Abhandlung über die Bewegungsgesetze mit „Phoranomica“. Sie wurde allerdings erst 30 Jahre nach seinem Tod 1689 publiziert.

Für Leibniz, der zur Verwunderung mancher Jungius überaus hochschätzte (AA VI.4: 266, 726), war die Frage nach der Bewegung besonders Anfang der 70er Jahre zentral, als er sich im Zuge der Vorbereitungen auf seinen Parisaufenthalt intensiv mit den Philosophiereformern Galileo Galilei, Pierre Gassendi, Christiaan Huygens und insbesondere Thomas Hobbes auseinandersetzte. Immer wieder gibt er zu dieser Zeit in seinen Entwürfen und Korrespondenzen zu erkennen, wie sehr er die verhinderte Lektüre der Phoranomica bedauere (GP VI: 143; AA II.1: 96). Dennoch waren ihm vermutlich bereits Grundgedanken aus diesem Werk durch ein zirkulierendes Specimen bekannt, bevor er dann Ende der 70er Jahre Jungius' Schriften persönlich in Hamburg in Augenschein nahm. Von Jungius dürfte daher auch schon Leibniz den Titel ,Phoronomie' in seinen frühen Arbeiten zur Philosophia naturalis entlehnt haben. Mit Rekurs auf Galileis Bestimmung der Geometrie als „Logicam Mathematicam“ nennt Leibniz sie eine „Logicam Physicam“, eine sozusagen kontuitive, d. i. anschauungsgebundene Logik. Die Logik der Mathematik, so Leibniz, werde in der Logik der Physik um die Wissenschaft von der Bewegung (,scientiam motationum“) als dem Grund unserer Anschauungskonstitution ergänzt, was die Verknüpfung der Geometrie mit den Begriffen Zeit, Kraft und Tätigkeit $[=$,tempore, vir, actione“ $]$ erfordere, um das Gesetz der Bewegung aufzufinden (AA VI.3: 532 f.; AA VI.2: 333).

Mit dieser Bestimmung der Phoronomie als einer Logik der Physik ist klar, dass die heutige Synonymität mit der Kinematik nur insofern zutreffend ist, als man sich - wie wohl auch wieder Max Planck (1916: 3) - auf die zitierte Bestimmung Kants besinnt. Für Jungius' und auch noch des jungen Leibniz' Verständnis der Phoronomie ist sie jedoch noch keineswegs zutreffend, da die Frage nach den die Bewegung verursachenden Kräften explizit miteinbezogen wird. Die angeführte vortheoretische Trennung des Wie und des Warum einer Bewegung, wie sie bei Kant zugrunde liegt und wie ich meine, sie auch etymologisch begründen zu können, ist ganz wesentlich erst das Ergebnis der leibnizschen Meditationen über die Einheit von Natur und Gnade, das heißt der modernen Wissenschaft mit der christlichen Pietas, die freilich als nichts anderes zu verstehen ist als die Einheit von Geistes- und Naturwissenschaft.

Die Forderung nach einer solchen Einheit verwebt Leibniz im Stile Platons in die Namensgebung und
Charaktere seines Dialogs über die Bewegung. Bemerkenswert ist dabei insbesondere dessen innerer Rahmen, der durch die Figuren Gallutius und Theophilius bestimmt wird. Gallutius ist Arzt und - so wird in versteckter Ironie betont - aufgrund seiner experimentellen Vorgehensweise stets erfolgreich. Theophilius wird als bedächtiger Greis vorgestellt, der eine erfolgreiche politischen Karriere hinter sich hat und (dennoch) seine Pietas, seine feste Gesinnung nicht verloren habe, wie es allenthalben von Kritikern der materialistischen Philosophie befürchtet wird. Theophilius verkörpert mithin das Reich des Geistes bzw. der Gnade; Gallutius hingegen ist vermeintlich reiner Analyst des Reichs der Natur. Gallutius lässt Leibniz zu Beginn noch die Überzeugung aussprechen, dass der Grund der Wissenschaft nur auf empirischen Wege gefunden werden könne (AA VI.3: 531). Nach Durchgang der Untersuchung aber hält ihm Theophilius entgegnen, dass die Wissenschaft nicht ohne die Theologie, d. h. nicht ohne höheren Prinzipien als den bloß geometrischen auskommen könne. Leibniz bringt diese Forderung auf die Formel ,philosophia cum pietas“ (AA VI.3: 570), die sich im Wunsch des Charinus' nach einer ,speculationem praxis connectat" (AA VI.3: 531) vom Beginn des Dialoges spiegelt.

Diese Einheit aus Wissenschaft und Theologie sah Leibniz insbesondere durch das hobbessche Postulat nach einer „doppelten Buchhaltung“ (Lotze 1852: 36) von Wissenschaft und Theologie bedroht (Hobbes 1915: 2535). Die Aufsuchung und mathematische Formulierung der Bewegungsgesetze wurde vom aufstrebenden Mechanizismus ja als nichts anderes verstanden als eben ihren Grund aufzuzeigen; und von Seiten der Theologie war für Hobbes und die ihm folgenden Materialisten eher wenig bzw. nur Irreführendes zu erwarten. Dass es aber etwas anderes ist, eine „reine Theorie“ (Stekeler-Weithofer 2018: 147) aufzustellen, die genaue Extrapolationen über zukünftige Ereignisse erlaubt, und diese selbst wiederum $\mathrm{zu}$ rationalisieren, das ist, wie ich meine, einer der wichtigsten Beiträge, die Leibniz zur angeblichen wissenschaftlichen Revolution des 16. und 17. Jahrhunderts beizutragen vermochte; und seine Überlegungen zur Rationalisierung der Bewegung spielen in seinen frühen Lebensjahren hierbei eine ganz entscheidende Rolle.

Leibniz machte seine erste Bekanntschaft mit den neuesten wissenschaftlichen Erkenntnissen aus Italien, Frankreich und England wohl bereits als Teenager während des Besuchs der Vorlesungen von Erhard Weigel $^{5}$ in Jena, wo ein deutlich frischerer Wind wehte als im eher konservativ-dogmatischen Leipzig in der Mitte des 17. Jahrhunderts Im Zuge dessen dürfte er daher alsbald auf das Problem aufmerksam geworden sein, das ihn fortan sein ganzes Leben lang begleitet hat: dass nämlich die fortschrittlichste Wissenschaft am meisten dazu neige, sich selbst zu überschätzen und im eigenen Erfolg die Rationalisierung ihrer Prinzipien bereits ausgemacht zu sehen. Dadurch übersehe sie allzu schnell die Symbolik, der sie sich bediene, und entkoppele sich so von ihren eigenen Grundlagen. Dass Leibniz diese

\footnotetext{
${ }^{5}$ Auch Weigel nutzte den Neologismus ,Phoronomie' in seiner Philosophia mathematica etc. (1693: 366).
} 
vornehmlich im religiösen Glauben ausmacht, darf nicht darüber hinweg täuschen, dass hiermit vor dem Hintergrund der modernen Wissenschaften die Einsicht in die Frage des wissenschaftlichen Selbstverständnisses formuliert war, wie sie für die folgenden drei Jahrhunderte insbesondere für die Philosophie in Deutschland bestimmend sein und sich vielfach wiederholen sollte. Einleitend $\mathrm{zu}$ einer kleinen Gelegenheitsschrift von 1668, die von ihrem Herausgeber mit Confessio Naturae contra Atheistas betitelt wurde, kommt dieses Paradox der Verdunkelung durch die Aufklärung ${ }^{6}$ im Rückblick auf diese Einsicht seiner Studienjahre zum ersten Mal in aller Deutlichkeit in folgenden Worten zum Ausdrucke:

\begin{abstract}
„Diese Tatsache aber, dass unsere Seele durch ihr eigenes Licht, d. h. die Philosophie, verdunkelt wird, ist mir stets besonders unwürdig erschienen.“ [= „Hoc vero mihi admodum indignum visum est animum nostrum sua ipsius luce, id est Philosophia praestringi."] (AA VI.1: 489)
\end{abstract}

Der hier zum Ausdruck kommende Anspruch des äußerst selbstbewussten 22-Jährigen ist nichts weniger als die Aufklärung der Aufklärung über die Notwendigkeit einer Rationalisierung ihrer eigenen Fundamente, die er selbst zu diesem Zeitpunkt lediglich in ihren Grundzügen kannte und wohl auch erst verstanden hatte. Abgesehen davon, dass er später selbst von dem atomistischen Standpunkt, von dem er in diesem Aufsatz ausgeht und sich überzeugt zeigt, wieder abgerückt ist, verdient seine Überlegung dennoch insofern Beachtung, als dass dort bereits ein Grundgedanke seiner späteren Monadologie ausgesprochen wird. Dieser lautet, dass die Körper, die nach Descartes' Bestimmung der substantia corporea [= körperliche Substanz] nichts anderes sind als räumliche Ausdehnung (AT VIII.1: 46), sich entgegen dessen Auffassung gerade nicht selbst genügen (AA VI.1: 490). Als wichtiges, ja entscheidendes Beispiel dient ihm die Bewegung, deren Grund nicht aus den primären Qualitäten der Körper bzw. des Ausgedehnten abgeleitet, mithin nicht innerhalb der scientia extensionis $[=\mathrm{Wis}-$ senschaft der Ausdehnung] bestimmt werden könne:

„Der Grund also für die Bewegung kann in
den sich selbst überlassenen Körpern nicht
aufgefunden werden.“ [= , Ratio igitur motus
in corporibus sibi relictis reperiri non
potest.“" (AA VI.1: 491)

Vielmehr sei es sogar gerade umgekehrt, so argumentiert er mit expliziten Rekurs auf Aristoteles wenig später in einem unbeantworteten Brief an den Kopf der Jansenisten in Frankreich Antoine Arnauld: Nicht die Bewegung ließe sich aus den spatialen Eigenschaften des Körpers geometrisch bestimmen, sondern die Substantialität des Körpers selbst [=,substantiam corporis seu naturam“ $]$ sei allein im Prinzip der Bewegung [=,principium motus"]

${ }^{6}$ Im 20. Jahrhundert firmierte dieses Paradox bekanntlich unter dem Titel einer „Dialektik der Aufklärung“ (Horkheimer \& Adorno 1969). auszumachen, das es für ein rationales Verständnis des Körpers zuvorderst zu ergründen gelte (AA II.1: 281).

Seine nähere Argumentation für diesen Befund, der ihm als Beweis der notwendigen Einheit von Physik und Theologie dient, lohnt es an dieser Stelle nicht weiter zu analysieren. Von Interesse aber ist der Impetus, mit dem Leibniz auftritt: Seine Argumentation zielt darauf ab, anhand einer Demonstration innerhalb des Rahmens einer rein physikalischen Betrachtung, diesen Rahmen selbst zu sprengen bzw. die Notwendigkeit seiner Überschreitung aufzuzeigen. Hierfür spricht etwa auch, dass er im eingangs zitierten Dialog über die Bewegung den Charinus selbst dieses Desiderat eines die Phoronomie rationalisierenden Übergangs von der Geometrie zur Physik [=,Transitum Geometria ad Physicam“, (AA VI.3: 532)] aussprechen lässt. Dieser Herangehensweise ist Leibniz stets treu geblieben und sie hat ihn auf einen Fehler der Cartesianer geführt, dessen Entdeckung für ihn zu einem Schlüsselereignis werden sollte, mit dem seine Monadologie anhebt.

\section{Dynamik oder Logik des Lebens}

Einen ersten großen Anlauf zur Rationalisierung der Bewegung unternahm Leibniz 1671 in seiner Abhandlung Theoria Motus Abstracti (AA 258-276), die er quasi als Gegenstück zu seiner Hypothesis Physica Nova / Theoria Motus Concreti (GP IV: 177-219) verfasst hat. In ihr beklagt er gleichfalls die bisher unaufgeklärte Dunkelheit um das Wesen der Bewegung und kritisiert namentlich die Untersuchungen des Aristoteles', Descartes' und Hobbes' als ungenügend und sogar irreführend im Hinblick auf eine abstrakte Behandlung. Trotz des ausdrücklichen Lobes für die Arbeiten Galileo Galileis und Honoratus Fabrius auf dem Gebiet der ,experimentellen Phoronomie“, die er auch eine „Phoronomia Empiricae“ nennt, kommt er (allerdings nicht ohne den oben bereits thematisierten Hinweis auf die ausstehende Besorgung der Arbeiten des Jungius') zu dem Befund, dass es an einer „elementaren Phoronomie“, die er auch eine „Phoronomia Universalis“ nennt, nach wie vor mangele (AA VI.2: 275, 336). Es sei nämlich etwas anderes, die bloß mechanisch-konkrete Beweglichkeit [=, mobilitatem"] zu bestimmen und ihre Gesetze aufzuzeigen und die Bewegung selbst [=,ipsum motum“] abstrakt bzw. rational zu begreifen (AA VI.1: 490, VI.2: 274). Richtig sei zwar, dass eine experimentelle Untersuchung auch ohne eine abstrakte Perspektive auskomme, damit aber bloß symbolisch bleibe und stets einer Rationalisierung, hier dem Nachweis des ultimativen Bewegungsgrundes [= „ultima ratio motus“] (GP IV: 469) bedürftig bleibe.

Diese hier getroffenen Unterscheidungen zwischen einer abstrakten und einer konkreten, einer rationalen und empirischen bzw. einer universalen und experimentellen Phoronomie ist ein Vorbote des leibnizschen Vorhabens zu einer „Nova Scientia Dynamica“ (GM VI: 235). Diesem leibnizschen Projekt verdanken wir unseren bürgerlichen Begriff der Dynamik, der sich im Gegensatz zu Phoronomie bekanntlich durchgesetzt hat und jenen Teilbereich der Mechanik bezeichnet, der die bewirkenden Kräfte behandelt. Dass Leibniz Mitte der 1680er 
Jahre mit dem Begriff der Phoronomie nicht mehr zufrieden ist, lässt sich etwa dem handschriftlichen Übersichtsplan Guilielmi Pacidii plus ultra etc. (AA VI.4: 673-677) zu seinem nie vollendeten Hauptwerk Scientia Generalis entnehmen. Auf die Kapitel Arithmetik, Algebra, Geometrie und Optik folgt dort der Titel: „De Arte Tornatoria et Motuum vestigiis sive Phoralogica [= Die Kunst der Tornation und der Bewegungsablauf oder die Phoralogie]“. Das letzte Wort dieses Titels hat Leibniz jedoch wieder durchgestrichen und ihn durch „Phorographie" ersetzt (AA VI.4: 675 f.), woraus sehr schön hervorgeht, dass es sich bei dem Inhalt dieses Kapitels für ihn weniger um die theoretische als die deskriptive Analyse der Bewegung handeln sollte. Auf das Kapitel der Phorographie sollte dann das Kapitel folgen, für das er den Titel „Dynamica seu de Motuum causa [= Dynamik oder die Ursache der Bewegung]" vorgesehen hatte. Unter einer phoronomischen Bewegung versteht Leibniz also erst hier, wie später dann auch Kant, die bloß geometrische Behandlung einer zeitlichen Folge der Veränderung unter Absehung der Qualitäten dessen, was sich bewegt - ich hatte dies eingangs das bloße Bewegt-Werden genannt. Unter einer dynamischen Bewegung hingegen versteht Leibniz in dezidierter Anlehnung an Platon jene Bewegung, in der ihre causa, die qua harmonischer Parallelität von Grund und Ursache die ratio der phoronomischen Bewegung ist, je mitbegriffen wird - ich hatte dies eingangs als die Selbstbewegung eingeführt.

Jene bereits angesprochene Entdeckung nun, die Leibniz maßgeblich den Weg zu seiner Dynamik wies, fällt in dieselbe Zeit und wurde 1686 in einem kleinen Aufsatz mit dem vielsagenden Titel Brevis demonstratio erroris memorabilis Cartesii [= Kurze Demonstration über einen merkwürdigen Fehler des Descartes'] (AA VI.4: 2027-2030) in der Leipziger Acta Eruditorum (1686: 161-163) sowie noch im selben Jahr in französischer Übersetzung in Pierre Bayles Nouvelles de la Republique des Lettres (1686: 996-999) veröffentlicht. Anhand einer simplen Beweisführung kann Leibniz darin belegen, dass Descartes unzutreffend die bewegende Kraft gleich der Bewegung setzt und sein Satz von der Erhaltung der Bewegungsmenge unter dieser Voraussetzung unzutreffend wäre. Denn die Bewegungsmenge, d. i. das Produkt aus Masse und Geschwindigkeit von zwei fallenden Körpern, deren jeweiliges Verhältnis von Masse und Fallhöhe zueinander umgekehrt proportional ist, ist entgegen der Forderung des Erhaltungssatzes nicht dieselbe, da sich ihre Geschwindigkeit nach den galileischen Fallgesetzen zum Quadrat beschleunigt. Der eigentlichen Schlussfolgerung aus dieser Entdeckung allerdings enthält sich Leibniz in diesem Aufsatz noch und belässt es zunächst bei der Demonstration dieses eigentümlichen Fehlers. Zeitgleich befand er sich nämlich in einem schon Anfang der 70er Jahre ersehnten Austausch mit dem ins Exil geflüchteten Arnauld, über seine neue Hypothese $\mathrm{zu}$ einem neuen metaphysischen System, das wir heute als die Monadologie kennen und maßgeblich auf dieser Entdeckung fußt. ${ }^{7}$ Von ihm er-

${ }^{7}$ Willy Kabitz (1909: 1-3) hat freilich vollkommen $\mathrm{zu}$ Recht konstatiert, dass die Monadologie keineswegs allein aus hoffte er sich wohl eine autoritäre Vorabbestätigung zu seinem neuen System, um sich für die zu erwartenden Auseinandersetzungen mit den Atomisten und Cartesianern zu wappnen. Diese blieb allerdings vorerst aus und die Korrespondenz war eher von gegenseitigen Missverständnissen geprägt. Leibniz blieb daher vielleicht auch aus diesem Grunde weiter zurückhaltend mit der Publikation seiner Meditationen.

Ein ganzes Jahrzehnt später, kurz nach dem Tod von Arnauld, spricht er dann erstmals in einer kleinen Ankündigungsschrift zu seinem Système nouveau mit dem Titel De primae philosophiae emendatione et de notione substantiae [= Über die Verbesserung der Ersten Philosophie und den Begriff der Substanz] (GP IV: 477487) öffentlich aus, was er meint, aus jenem konstatierten Fehler der Cartesianer schließen zu können:

„Um davon [sc. der Verbesserung der Philosophie] einen Vorgeschmack zu geben, will ich vorerst sagen, dass der Begriff der Kräfte, oder des Vermögens (den die Deutschen Krafft [sic!], die Franzosen la force nennen), für dessen Erklärung ich im Besonderen die Wissenschaft der Dynamik bestimmt habe, sehr viel Licht zur Erkenntnis des wahren Begriffs der Substanz beiträgt.“ $[=$, ,Cujus rei ut aliquem gustum dem, dicam interim, notionem virium seu virtutis (quam Germani vocant Krafft, Galli la force) cui ego explicandae peculiarem Dynamices scientiam destinavi, plurimum lucis afferre ad veram notionem substantiae intelligendam."] (GP IV: 469)

Die erwähnte Vorsicht, mit der Leibniz seine Überlegungen präsentierte, erklärt sich aus dieser Besinnung auf den Kraftbegriff, der zu jener Zeit und auch noch in den folgenden Jahrhunderten stets den Beigeschmack der qualitates occultae trug. ${ }^{8}$ Er folgert ihn daraus, dass der

Leibniz' Dynamik interpretiert werden darf, wie dies etwa Eduard Dillmann (1891) unternommen hat. Dies würde selbstverständlich die zweite tragende Säule, die leibnizsche Logik, insbesondere die Arbeiten zur ars inveniendi ignorieren, wie die Arbeiten Betrand Russells (1900) und Louis Couturat (1901) nachgewiesen haben. Dennoch wird hiermit klar, welche große Bedeutung die Überlegungen zu einer neuen Wissenschaft der Dynamik für Leibniz' reifes philosophisches System haben.

${ }^{8}$ Die Skepsis gegenüber dem Kraftbegriff hallt auch noch in David Humes Enquiry Concerning Human Understanding VII.2 (2007: 86) nach, wenn es dort heißt: „There are no ideas, which occur in metaphysics, more obscure and uncertain, than those of power, force, energy or necessary connexion [...].“ Ähnlich äußert sich dann auch wieder Heinrich Hertz, der den Kraftbegriff im Vorwort zu seinen Prinzipien der Mechanik (1894: 14) als Beispiel eines jener metaphysischen Begriffe anführt, die er als mitlaufende, aber eigentlich „leergehende Nebenräder" der wissenschaftlichen Modellierung der Wirklichkeit versteht und die er zur Förderung der Ruhe einer von der Metaphysik gequälten Wissenschaftlerseele aus dem wissenschaftlichen Vokabular gerne endgültig eliminiert sähe. Eine Metapher, die im Übrigen nicht unerheblich für die durch Ludwig Wittgenstein geprägte Philosophie des 20. Jahrhundert 
Brevis demonstratio zufolge dem Erhaltungssatz nicht die cartesische „quantitas motus“ [= Bewegungsmenge] - die Leibniz auch ,vis mortis“ [= tote Kraft] nennt - zugrunde liegt, sondern allein ein ,principium activum“ [= Prinzip der Aktivität] aus dem Produkt von Masse und Geschwindigkeit zum Quadrat, das er in primitiver, das heißt metaphysischer Hinsicht auch die „vis viva“ [= lebendige Kraft] in der Natur nennt. Dieses Prinzip habe zwar schon Galilei in seiner experimentellen Phoronomie entdeckt, ohne sich aber ihrer Bedeutung bewusst geworden zu sein. So heißt es etwa im Specimen Dynamicum etc. I, $\S 8$ :

„Galilei begann von der Kraft des Lebens (wenn auch noch unter anderem Namen, dennoch dem Begriff nach) zu handeln.“ $[=$,Galilaeus de vi viva (alio licet nomine, imo conceptu) agere coepit."] (GM VI: 239, 242 f. $)^{9}$

Für Leibniz ist damit klar, dass es einer neuen Disziplin bedarf, die sich eigens diesen Prinzipien des Lebens [=,les principes de vie“] (GP VI: 539-546) annehmen müsse: der Dynamik. Erst durch eine solche Wissenschaft werde verständlich, dass die Lebewesen nicht bloß als aufgezogene Uhren, sondern mit Perzeptionen und Apperzeptionen begabte Vollzugssubjekte in der Welt und im Falle von jenen, die Wissenschaft betreiben, auch im Hinblick ihre Bezüge auf die Welt begriffen werden müssen.

Die ,große Bedeutung [ [= „magni momenti“, ,,assez importante"], die Leibniz dieser Entdeckung beimaß, lässt sich bereits daraus ersehen, dass er diesen Aufsatz quasi in sämtlichen nachfolgenden Schriften mehr oder weniger explizit immer wieder selbst zitierte und gegen die Cartesianer und Okkasionalisten ins Feld führte. ${ }^{10}$ Leibniz ist überzeugt, damit endlich den entscheidenden Nachweis gefunden zu haben, den er erfolglos bereits in der Confessio Naturae contra Atheistas versucht hatte zu führen: nämlich allein aus den Annahme der geometrischen Prinzipien der scientia extensionis selbst zu demonstrieren, dass sich die Körper nicht genügen und sich daher aus ihnen allein die Bewegung niemals wird rationalisieren lassen können. Er behauptet später sogar,

\footnotetext{
wurde (Borchers 2014).

${ }^{9}$ Allerdings ist sich Galilei des initialen Charakters seiner Arbeiten mit Blick auf eine erst noch auszuarbeitenden Disziplin durchaus sehr bewusst, wenn er im Discorsi (Ed. Naz. VIII: 190) bekennt, dass die eigentlichen Folgerungen aus seinen Einsichten größeren Denkern als ihm überlassen blieben. Unbezweifelbar hat Leibniz diese Bemerkung als Aufforderung verstanden und sah sich eben genau dieser Aufgabe gegenüber.

${ }^{10}$ Leibniz zitiert ihn fast vollständig im etwa zeitgleich verfassten Discours de Métaphysique § 17 (AA VI.4: 1556-1558) und im Brief an Arnauld vom 14. Juli 1686 (AA II.2: 83 f.); ausführliche Erwähnung findet er weiter in Animadversiones etc. II, § 36 (GP IV: 370-372), Specimen Dynamicum etc. I, $\S 17$ (GM VI: 245 f.), Eclaircissement du nouveau système de la communication des substances etc. (GP IV: $497 \mathrm{f}$.) sowie in der Essais de Théodicée etc. III, § 345 (GP VI: 319) und den Principes de la Nature et de la Grâce Fondés en Raison $§ 11$ (GP VI: 603), um nur die bekanntesten Beispiele zu nennen.
}

dass wenn Descartes den konstatierten Fehler in seiner Bestimmung des Kraftbegriffs selbst schon bemerkt hätte, ihn diese Entdeckung den Weg zu seinem Begriff der Substanz als Monade bereits gewiesen hätte. ${ }^{11}$ Die substantia corporea erschöpfe sich nach Leibniz hiernach

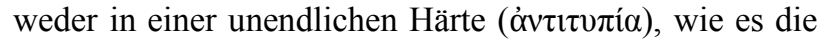
Annahme der Atomisten, noch im Begriff der räumlichen Ausdehnung, wie es die Auffassung der cartesianischen Korpuskalarphilosophie war, sondern allein in einem lebendigen Prinzip, das Leibniz mit Rekurs auf Aristote-

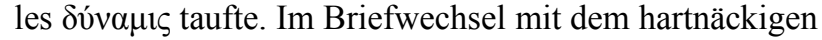
Cartesianer Burchard de Volder konstatiert Leibniz hierzu am 24. März / 3. April 1699:
„Ich glaube daher, dass unser Denken erst im Begriff $\tau \tilde{\omega} v \delta v v \alpha \mu \iota \kappa \tilde{\omega} v$, und nicht in dem der Ausdehnung zur Vollendung und zur Ruhe kommt." $[=$,Itaque magis in notione $\tau \tilde{\omega} v \delta v v \alpha \mu 1 \kappa \tilde{\omega} v$ quam extensionis cogita- tionem nostram compleri et terminari credo.00"] (AA II.3: 546)

In Anlehnung an Leibniz' frühere Bestimmung der Phoronomie als Logik der Physik ist es daher vielleicht erlaubt, die Dynamik im leibnizschen Sinne eine „Logik des Lebens" (Wilson 1994) zu nennen, die ihrerseits freilich nach einer Rationalisierung verlangt, die Leibniz sodann in seinem System der ,lebendigen Spiegel ${ }^{\circ}$ (GP VI: 599), seiner Monadologie unternommen hat.

\section{Schluss}

Selbstverständlich würde es uns heute nicht mehr einfallen, das Prinzip des Lebens auf eine simple Demonstration der Beschleunigung durch Gravitation zurück zu führen. Gleichwohl gelingt Leibniz hier die zutreffende Unterscheidung von Impuls und Kraft, was den Cartesianern noch nicht gelungen war. Insofern ist seine Meditation über die Bewegung und seine Brevis demonstratio als Ausgangspunkt der Dynamik zunächst von wissenschaftshistorischem Interesse. Doch gerade aufgrund der Simplizität ist seine Demonstration auch ein schillerndes Beispiel für die Ignoranz szientistischer Theoriebildungen. Worauf Leibniz die Cartesianer nämlich in epistemologischer Perspektive stets zurecht hinweist, das ist ihre Überschätzung reiner Theorien als zutreffende Modellierungen von Weltereignissen.

$\mathrm{Zu}$ beachten gilt es stets, dass Theorien von Vollzugssubjekten in der Welt erfunden werden, insofern lokal sind - und bleiben. Leibniz weist mithin ganz zu Recht darauf hin, dass Descartes an seinem eigenen Anspruch scheitert. Zwar kündigt er einerseits das Erkenntnissubjekt als einzigen Ausgangspunkt seines Systems

${ }^{11}$ Deutlich wird dies etwa im Brief an Arnauld vom 14 Juli 1686 (AA II.2: 58), im Système nouveau etc. $§ 12$ (GP IV: 483), Considération sur les principes de vie etc. (GP IV: 540), Essais de Théodicée etc. I, § 61 (GP VI: 136), Monadologie $\S 80$ (GP VI: $620 \mathrm{f}$.) und im Brief an Remond vom 10. Jan. 1714 (GP III: 607). Bemerkenswert ist eine diesbezügliche Andeutung bereits Ende der 1670er Jahre in De numeris characteristicis ad linguam universalem constituendam (AA VI.4: 267). 
an und erkennt damit richtig das Primat des lokalen Vollzugs für jede Geltung von Wissensansprüchen. Andererseits, so Leibniz' Vorwurf, übergeht er selbst allzu voreilig seine eigene Einsicht, indem er den Gegenstandsbereich der Wissenschaft auf die spatiale Extension beschränkt. $^{12}$ Leibniz entlarvt also gerade mit seinem Rekurs auf traditionelle und vermeintlich $\mathrm{zu}$ überwindende Einsichten den Anspruch auf eine globale Gottesperspektive als den modernen Aberglauben der wissenschaftlichen Revolution und Aufklärung.

\section{Literatur}

Aristoteles (1987) Metaphysik, Bd. I: Bücher I(A)zVI(E), Bd. II: Bücher VII(Z)-XIV(N), übers. v. H. Bonitz, hg. v. H. Seidl, Hamburg.

Aristoteles (1987) Physik, Bd. I: Bücher I(A)-IV( $\Delta)$, Bd. II: Bücher $\mathrm{V}(\mathrm{E})-\mathrm{VIII}(\Theta)$, übers. u. hg. v. H. G. Zekl, Hamburg.

Augustinus (2009) Bekenntnisse [Confessiones], übers. u. hg. v. K. Flasch \& B. Mojsisch, Stuttgart.

Borchers, R. (2014) „Leerlaufende Räder und TLP 6.53“, S. 36-39 in: Analytic and Continental Philosophy: Methods and Perspectives, ed. by S. Rinofner-Kreidl and $\mathrm{H}$. A. Wiltsche, Kirchberg am Wechsel.

Couturat, L. (1901) La logique de Leibniz d'apèes des documents inédits, Paris.

Bacon F. (1999) Novum Organum (1620), Bd. I, eingl. u. hg. v. W. Krohn, Hamburg.

Descartes, R. (1897-1910) OEuvres de Descartes, Bd. IXII, hg. v. C. Adam \& P. Tannery, Paris (Sigle: AT).

Dillmann, E. (1891) Eine neue Darstellung der Leibnizischen Monadenlehre auf Grund der Quellen, Norderstedt.

Galilei, G. (1890-1909) Edizione nazionale sotto gli auspicii di Sua Maestà il Re d'Italia, Bd. VI \& VIII, hg. v. A. Favaro, Firenze (Sigle: Ed. Naz.).

Gehlers, J. S. T. (1833) Physikalisches Wörterbuch, Bd. 7, Leipzig.

Hermann, J. (1716) Phoronomia, sive de viribus et motibus solidorum etc., Amsterdam.

Hertz, H. (1894) Die Prinzipien der Mechanik im neuen Zusammenhange dargestellt, Bd. 3 der Gesammelten Werke, hg. v. Ph. Lenard, Leipzig.

Hobbes, Th. (1651) Leviathan or the Matter, Forme and Power of A Commonwealth Ecclesiasticall and Civil, London.

Hobbes, Th. (1914) Elemente der Philosophie Teil I: Vom Körper [ Elementorum philosophiae sectio prima: De corpore (1655)], übers. u. hg. v. M. Frischeisen-Köhler Berlin.
Horkheimer, M. \& Adorno, T. H. W. (1969) Dialektik der Aufklärung. Philosophische Fragmente, Frankfurt a. M.

Hume, D. (2007) Eine Untersuchung über den menschlichen Verstand [ An Enquiry Concerning Human Understanding (1748)], Frankfurt a. M.

Jungius, J. (1689) Phoranomica, seu Doctrina de motu locali etc., Hamburg.

Kabitz, W. (1909) Die Philosophie des jungen Leibniz, Hildesheim.

Kant, I.(1902 ff.) Gesammelte Schriften Bd. IV, hg. v. der Preussischen Akademie der Wissenschaften, Berlin (Sigle: AA).

Kirchner, F. \& Michaëlis, C. (1998) Wörterbuch der philosophischen Begriffe, weitergeführt von J. Hoffmeister, vollst. neu hg. v. A. Regenbogen \& U. Meyer, Hamburg.

Leibniz, G. W. (1906) Nachgelassene Schriften physikalischen, mechanischen und technischen Inhalts, hg. v. E. Gerland, Leipzig.

Leibniz, G. W. (1930 ff.) Sämtliche Schriften und Briefe, Reihe II: Philosophischer Briefwechsel, $6 \mathrm{Bd}$. und Reihe VI: Philosophische Schriften 6 Bd., hg. v. der BerlinBrandenburgischen Akademie der Wissenschaften und der Akademie der Wissenschaften in Göttingen, Berlin (Sigle: AA).

Leibniz, G. W. (1849-1890) Philosophische Schriften, Bd. I-VII, hg. v. C. I. Gerhardt, Berlin (Sigle: GP).

Leibniz, G. W. (1849-1863) Mathematische Schriften, Bd. I-VII, hg. v. C. I. Gerhardt, Berlin (Sigle: GM).

Lotze, R. H. (1852) Medicinische Psychologie oder Physiologie der Seele, Leipzig.

Mainzer, K. (1984) Artikel „Phoronomie“, S. 931 f. in: J. Ritter / K. Gründer / G. Gabriel (Hg.), Historisches Wörterbuch der Philosophie (Bd. VII), Basel.

Planck, M. (1916) Einführung in die Allgemeine Mechanik, Leipzig.

Platon (2016) Werke, Bd. I-VIII, übers. v. F. Schleiermacher, bearb. v. H. Hofmann, Darmstadt.

Russell, B. (1900) A Critical Exposition of the Philosophy of Leibniz, Cambridge.

Stekeler-Weithofer, P. (2018) Kritik der reinen Theorie. Logische Differenzen zwischen Wissenschaft und Weltanschauung, Tübingen.

Thomas v. Aquin (2018) Opera omnia, hg. v. der Universidad de Navarra (www.corpusthomisticum.org).

Weigel, E. (1693) Philosophia mathematica etc., Jena.

Wilson, C. (1994) „Leibniz and the Logic of Life“, Revue internationale de philosophie 48/188: 237-253.

\footnotetext{
${ }^{12}$ Vgl. hierzu Leibniz' sehr treffenden Vergleich von Descartes mit dem antiken Philosophen Anaxagoras in Platons Dialog Phaedon (97b-99c) im Discours de Métaphysique $\S 20$ (AA VI.4: 1562 f.).
} 